\title{
New emerging results on molecular gas, stars, and dust at $z \sim 2$, as revealed by low star formation rate and low stellar mass star-forming galaxies
}

\author{
Miroslava Dessauges-Zavadsky ${ }^{1}$, Michel Zamojski ${ }^{1}$, Daniel Schaerer $^{1}$, \\ Françoise Combes ${ }^{2}$, Eiichi Egami ${ }^{3}$, Panos Sklias ${ }^{1}$, \\ Mark A. Swinbank ${ }^{4}$, Johan Richard ${ }^{5}$ and Tim Rawle ${ }^{6}$ \\ ${ }^{1}$ Observatoire de Genève, Université de Genève, \\ 51 Chemin des Maillettes, 1290 Versoix, Switzerland \\ ${ }^{2}$ Observatoire de Paris, LERMA, \\ 61 Avenue de l'Observatoire, 75014 Paris, France \\ ${ }^{3}$ Steward Observatory, University of Arizona, \\ 933 North Cherry Avenue, Tucson, AZ 85721, United States \\ ${ }^{4}$ Institute for Computational Cosmology, Durham University, \\ South Road, Durham DH1 3LE, United Kingdom \\ ${ }^{5}$ CRAL, Observatoire de Lyon, Université Lyon 1, \\ 9 Avenue Ch. André, 69561 Saint Genis Laval Cedex, France \\ ${ }^{6}$ ESAC, ESA, PO Box 78, Villanueva de la Canada, 28691 Madrid, Spain
}

\begin{abstract}
Recent CO surveys of star-forming galaxies (SFGs) at $z \sim 2$ have revolutionized our picture of massive galaxies. It is time to expand these studies toward the more common $z \sim 2$ SFGs with SFR $<40 \mathrm{M}_{\odot} \mathrm{yr}^{-1}$ and $M_{*}<2.5 \times 10^{10} \mathrm{M}_{\odot}$. We have derived molecular gas, stars, and dust in 8 such lensed SFGs. They extend the $L_{\mathrm{IR}}-L_{\mathrm{CO}(1-0)}^{\prime}$ distribution of massive $z>1$ SFGs and increase the spread of the SFG star formation efficiency (SFE). A single star formation relation is found when combining all existing CO-detected galaxies. Our low- $M_{*}$ SFGs also reveal a SFE decrease with $M_{*}$ as found locally. A rise of the molecular gas fraction $\left(f_{\text {gas }}\right)$ with redshift is observed up to $z \sim 1.6$, but it severely flattens toward higher redshifts. We provide the first insight into the $f_{\text {gas }}$ upturn at the low- $M_{*}$ end $10^{9.4}<M_{*} / \mathrm{M}_{\odot}<10^{10}$ reaching $f_{\text {gas }} \sim 0.7$, it is followed by a $f_{\text {gas }}$ decrease toward higher $M_{*}$. Finally, we find a non-universal dust-to-gas ratio among local and high-redshift SFGs and starbursts with near-solar metallicities.
\end{abstract}

Keywords. Gravitational lensing, galaxies: evolution, galaxies: high-redshift

\section{Introduction}

In the local Universe, the properties of the molecular gas, the fuel for star formation, is routinely probed using the rotational transitions of CO. Nevertheless, at high redshift CO measurements are challenging and have so far only been possible in "main sequence" (MS) star-forming galaxies at $1<z<2.5$ distributed at the high star formation rate (SFR) and high stellar mass $\left(M_{*}\right)$ end. The probe of the molecular gas in the more common and numerous MS galaxies characterised by IR luminosities below the characteristic $L^{\star}=4 \times 10^{10} \mathrm{~L}_{\odot}$ luminosity of the IR luminosity function (Gruppioni et al. 2013) is essential, since galaxies following the MS between $M_{*}$ and SFR contribute all together $\sim 90 \%$ to the cosmic SFR density at $1<z<3$ (Daddi et al. 2007; Rodighiero et al. 

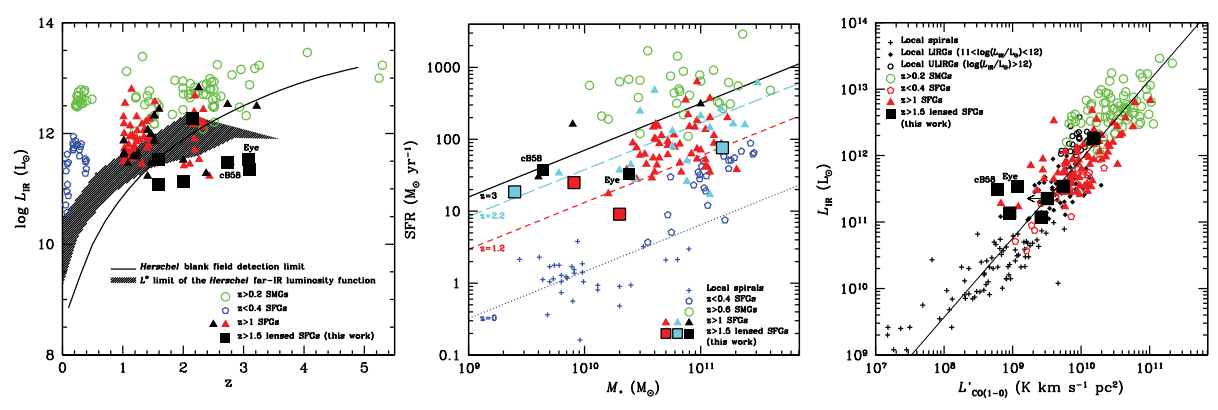

Figure 1. Comparison of the lensed, sub- $L^{\star}$ SFGs with our compilation of CO-detected galaxies from the literature (see Dessauges-Zavadsky et al. 2015). IR luminosities as a function of redshift (left), star formation rates as a function of stellar masses with the color-coding referring to 3 redshift bins (middle), and IR luminosities as a function of $\mathrm{CO}(1-0)$ luminosities (right).

2011). CO measurements in sub- $L^{\star}$ SFGs at $z>1$ can be achieved with the help of strong gravitational lensing.

\section{Target selection}

We have selected five targets for new IRAM/PdBI and IRAM/30m CO observations from the Herschel Lensing Survey (HLS; Egami et al. 2010). HLS is a survey of 54 massive galaxy clusters aimed at identifying cluster-lensed, high-redshift background sources. We have applied the following selection criteria to the CO follow-up galaxies: spectroscopic redshifts $z \sim 1.5-3$; lensing-corrected Herschel IR luminosities $L_{\mathrm{IR}}<L^{\star}$, or equivalently $\mathrm{SFR}<40 \mathrm{M}_{\odot} \mathrm{yr}^{-1}$; high magnification factors $\mu>10$ to achieve CO detections in these intrinsically low- $L_{\mathrm{IR}}$ SFGs; and complete photometry from optical, near-IR, IR to far-IR to enable to derive the stellar and dust properties from the spectral energy distribution (SED) modeling. We add to this sample the two well known strongly-lensed galaxies, MS1512-cB58 and the Cosmic Eye, that also satisfy the above selection criteria.

\section{Comparison of the sub- $L^{\star}$ SFGs with other CO-detected galaxies}

\subsection{IR luminosities, stellar masses, and CO luminosities}

When comparing the selected cluster-lensed, sub- $L^{\star}$ SFGs with our compilation of galaxies with CO measurements from the literature (see Dessauges-Zavadsky et al. 2015 for the references), we show in Fig. 1 (left panel) that they probe the lower $L_{\mathrm{IR}}$ among all CO-detected galaxies at $z>1.5$ and even fall below the Herschel blank field detection limit (Elbaz et al. 2011; Sklias et al. 2014). Despite their intrinsically low SFR, they lie on the MS at their respective redshifts, but have one order of magnitude smaller $M_{*}$ with $M_{*}<2.5 \times 10^{10} \mathrm{M}_{\odot}$ (Fig. 1, middle panel). Thus, our sub- $L^{\star}$ SFGs sample a new $L_{\mathrm{IR}}$, $M_{*}$ parameter space of galaxies with CO detections. When plotting IR luminosities as a function of $\mathrm{CO}(1-0)$ luminosities, we see that the sub- $L^{\star}$ SFGs populate the same domain as the local luminous-IR galaxies (LIRGs), their $L_{\mathrm{IR}}$ counterparts at $z=0$, and perfectly extend the observed $L_{\mathrm{IR}}-L_{\mathrm{CO}(1-0)}^{\prime}$ distribution of previously studied $z>1$ SFGs with higher $L_{\mathrm{IR}}$ (Fig. 1, right panel). They also contribute to increase the spread of the star formation efficiency, $\mathrm{SFE}=L_{\mathrm{IR}} / L_{\mathrm{CO}(1-0)}^{\prime} \equiv \mathrm{SFR} / M_{\text {gas }}$, of the $z>1 \mathrm{SFGs}$, since at a given $L_{\mathrm{IR}}$ one observes SFGs with lower $L_{\mathrm{CO}(1-0)}^{\prime}$. As a result, the high-redshift SFGs do not have their SFE confined to the low values of local spirals and the high-redshift submm galaxies do not systematically appear with an excess in SFE any more. This yields 

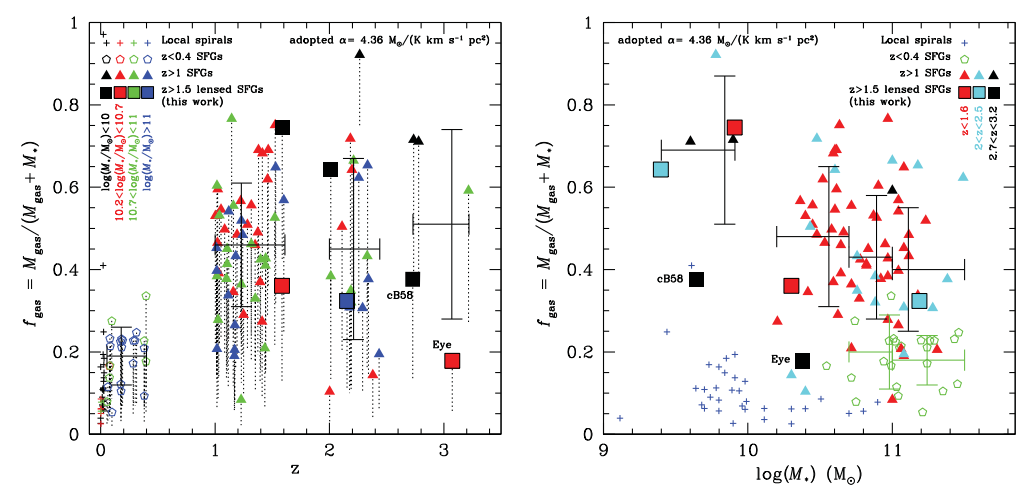

Figure 2. Left: Molecular gas fractions as a function of redshift with the color-coding referring to $4 M_{*}$ bins. A net $f_{\text {gas }}$ rise is observed from $z=0$ to $z \sim 1.6$. Right: Molecular gas fractions as a function of stellar masses with the color-coding referring to 3 redshift bins. A clear $f_{\text {gas }}$ upturn is found at the low- $M_{*}$ end.

a clear evidence for a single linear $\log \left(L_{\mathrm{IR}}\right)-\log \left(L_{\mathrm{CO}(1-0)}^{\prime}\right)$ relation for galaxies gathering together spirals, MS SFGs, and starbursts at redshifts from $z=0$ to 5.3 and sampling 5 orders of magnitude in $L_{\mathrm{IR}}$. A universal star formation hence is supported. The sub- $L^{\star}$ SFGs with low $M_{*}$ also trigger the SFE- $M_{*}$ anti-correlation at $z>1$, as observed in local galaxies (Saintonge et al. 2011; Bothwell et al. 2014). If correct, it strongly questions the constant molecular gas depletion timescale, $t_{\mathrm{depl}}=1 / \mathrm{SFE}$, of $0.7 \mathrm{Gyr}$ found by Tacconi et al. (2013) and acknowledged in bathtub models.

\subsection{Molecular gas fractions}

Various physical processes at play in the evolution of galaxies (accretion, star formation, and feedback) have direct impact on the molecular gas fraction $\left(f_{\text {gas }}\right)$. Therefore, $f_{\text {gas }}$ provides important tests of galaxy evolution models. Theoretically, it is accepted that $f_{\text {gas }}$ steadily increases with the Hubble time as a result of the expansion of the Universe (Obreschkow\& Rawlings 2009; Lagos et al. 2014). Observationally, we do observe a net rise of $f_{\text {gas }}$ from local spirals to CO-detected SFGs at $z \sim 1.6$, but this rise is followed by a mild, quasi non-existent increase toward higher redshifts (Fig. 2, left panel). This $f_{\text {gas }}$ flattening is also observed when $f_{\text {gas }}$ is derived from the far-IR dust continuum (Troncoso et al. 2014; Béthermin et al. 2015). Nevertheless, currently the observed redshift evolution of $f_{\text {gas }}$ is very tentative because of the few CO measurements (5) available beyond $z \sim 2.5$. The additional difficulty which enters into the probe of the cosmic evolution of $f_{\text {gas }}$ is the inherent large $f_{\text {gas }}$ dispersion per redshift bin. It is due to the strong dependence of $f_{\text {gas }}$ on $M_{*}$, such that galaxies with the smaller $M_{*}$ have the larger $f_{\text {gas }}$. This is illustrated by the color-coding of the data points referring to 4 different $M_{*}$ bins.

Models predict a drop in $f_{\text {gas }}$ with increasing $M_{*}$ and an $f_{\text {gas }}$ upturn at the low- $M_{*}$ end which strength depends on the adopted outflow (Bouché et al. 2010; Davé et al. 2011). Our sample of sub- $L^{\star}$ SFGs more than doubles the number of SFGs at $z>1$ (3 out of 5 ) with achieved $f_{\text {gas }}$ measurements at the low- $M_{*}$ end $10^{9.4}<M_{*} / \mathrm{M}_{\odot}<10^{10}$ and provides the first insight into the $f_{\text {gas }}$ upturn with $\left\langle f_{\text {gas }}\right\rangle=0.69 \pm 0.18$ (Fig. 2, right panel). SFGs with $M_{*} / \mathrm{M}_{\odot}>10^{10.4}$ show a mild decrease of $f_{\text {gas }}$, with again a large dispersion due this time to the $f_{\text {gas }}$ dependence on redshift, well highlighted by the $z<0.4$ SFGs.

\subsection{Dust-to-gas ratios}

Scoville et al. (2014) proposed to consider the rest-frame $850 \mu \mathrm{m}$ continuum as the dust mass tracer. They then derived in a homogeneous way (same Galactic $\mathrm{CO}-\mathrm{H}_{2}$ conversion 


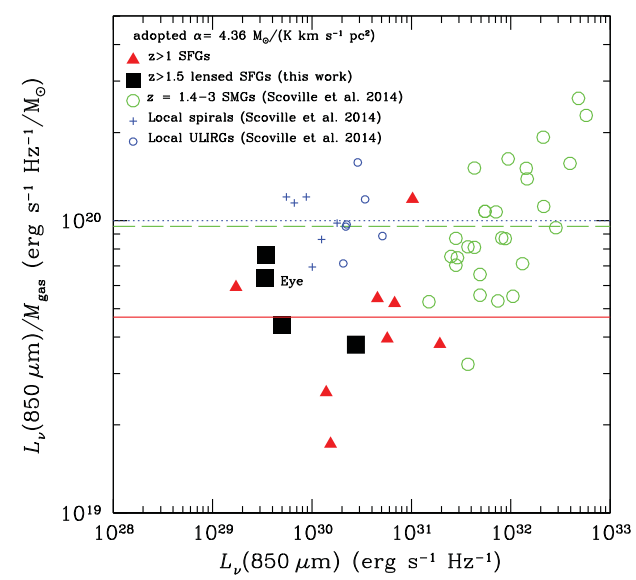

Figure 3. Dust-to-gas ratios estimated from the $850 \mu \mathrm{m}$ continuum over the molecular gas mass ratio following Scoville et al. (2014) prescriptions. Local spirals, local ULIRGs, high-redshift sub-mm galaxies, and high-redshift SFGs at fixed about solar metallicity are compared.

factor and $\beta$-slope $=1.8$ ) the dust-to-gas ratios (DGR) in local galaxies (spirals and ULIRGs) and $z>1.4$ sub-mm galaxies and found the same DGR means. They hence suggested that the dust continuum can also be used as a tracer of the molecular gas mass. We have applied the same prescriptions to estimate the DGR of our sample of lensed, sub- $L^{\star}$ SFGs plus a few $z>1$ SFGs from the literature for which the far-IR photometry has been published (Magdis et al. 2012; Saintonge et al. 2013). But, we consider only objects with about solar metallicities $\left(Z / Z_{\odot}>0.8\right)$, as derived from nebular emission line metallicity estimators or the mass-metallicity relation. A clear trend for a lower DGR mean in $z>1$ SFGs by $\sim 0.33$ dex at fixed about solar metallicity is observed, namely a factor of 2 difference with the DGR measured by Scoville et al. in local galaxies and highredshift sub-mm galaxies (see Fig. 3). This questions the universality of the DGR and puts strong warning on the use of the dust continuum as a molecular gas mass tracer. CO measurements should still be preferred despite the uncertain $\mathrm{CO}-\mathrm{H}_{2}$ conversion factor.

\section{References}

Béthermin, M., Daddi, E., Magdis, G., et al. 2015, A\&A, 573, 113

Bouché, N., Dekel, A., Genzel, R., et al. 2010, ApJ, 718, 1001

Bothwell, M. S., Wagg, J., Cicone, C., et al. 2014, MNRAS, 445, 2599

Daddi, E., Dickinson, M., Morrison, G., et al. 2007, ApJ, 670, 156

Dessauges-Zavadsky, M., Zamojski, M., Schaerer, D., et al. 2015, A\& A, 577, 50

Davé, R., Finlator, K., \& Oppenheimer, B. 2011, MNRAS, 416, 1354

Egami, E., Rex, M., Rawle, T. D., et al. 2010, $A \mathscr{E} A$, 518, 12

Elbaz, D., Dickinson, M., Hwang, H. S., et al. 2011, A\&A, 533, 119

Gruppioni, C., Pozzi, F., Rodighiero, G., et al. 2013, MNRAS, 432, 23

Lagos, C. d. P., Baugh, C. M., Zwaan, M. A., et al. 2014, MNRAS, 440, 920

Obreschkow, D. \& Rawlings, S. 2009, ApJL, 696, L129

Magdis, G. E., Daddi, E., Béthermin, M., et al. 2012, ApJ, 760, 6

Rodighiero, G., Daddi, E., Baronchelli, I., et al. 2011, ApJL, 739, L40

Saintonge, A., Kauffmann, G., Wang, J., et al. 2011, MNRAS, 415, 61

Saintonge, A., Lutz, D., Genzel, R., et al. 2013, ApJ, 778, 2

Scoville, N., Aussel, H., Sheth, K., et al. 2014, ApJ, 783, 84

Sklias, P., Zamojski, M., Schaerer, D., et al. 2014, A\&SA, 561, 149

Tacconi, L. J., Neri, R., Genzel, R., et al. 2013, ApJ, 768, 74

Troncoso, P., Maiolino, R., Sommariva, V., et al. 2014, A\&A A, 563, 58 